\title{
CALIBRATION OF MK TYPES BY FITTING \\ THE HR DIAGRAMS OF THREE MOVING CLUSTERS
}

\author{
R. F. GARRISON
}

David Dunlap Observatory, University of Toronto, Canada

Abstract. A composite HR diagram constructed from the main sequences of three moving clusters (Hyades, $\alpha$ Persei, and the Inner Region of Upper Scorpius) using MK classifications, is presented and discussed.

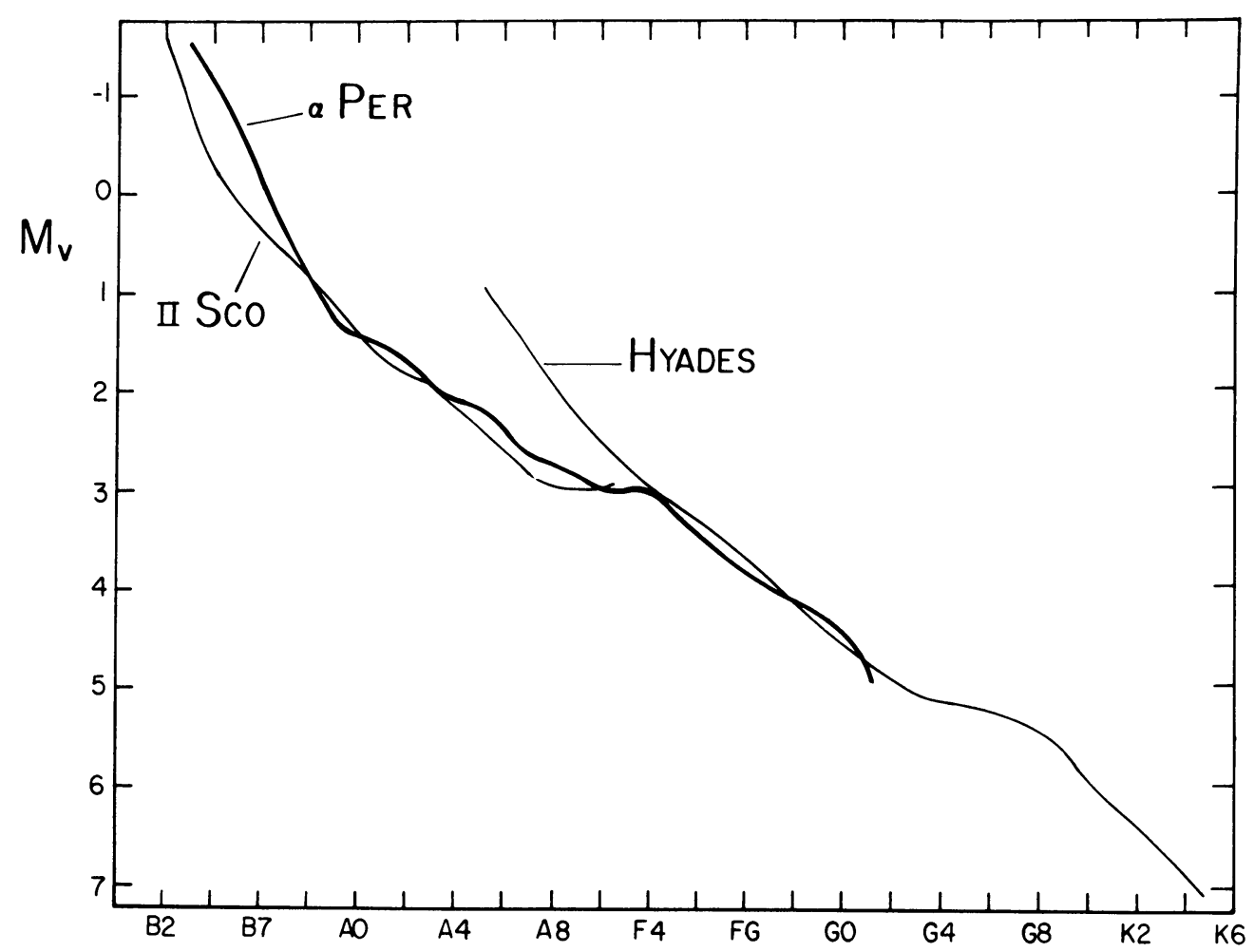

Fig. 1. Composite HR diagram for three moving clusters. The sequence labelled II Sco is for the Inner Region of Upper Scorpius.

Several years ago, Hiltner, Schild and I began a photometric $(U B V)$ and spectroscopic (MK) study of galactic structure in the southern hemisphere. We have now completed the observations for all the $\mathrm{OB}$ stars south of $-30^{\circ}$ and brighter than tenth magnitude which are listed in the Heidelberg objective prism catalogue (Klare and Szeidl, 1966).

Before these data can be used to study spiral structure, it is necessary to choose an absolute magnitude calibration. As a preliminary step, it is interesting to use the 
cluster-fitting procedure for clusters whose distance moduli can be independently determined and for which reliable MK classifications are available. Three clusters which fit these criteria are the Hyades, the $\alpha$ Persei cluster and the Inner Region of Upper Scorpius. The reason for choosing the Inner Region of Upper Scorpius instead of the entire Scorpio-Centaurus Association is that the former has a well-determined, narrow main sequence extending to F0. It is fortunate that the $\alpha$ Persei cluster bridges the age gap between the Hyades and Scorpius and provides a good overlap with both main sequences.

The MK types for the Hyades are from Morgan and Hiltner (1965); for the $\alpha$ Persei cluster, they are from Morgan et al. (1971); and for the Inner Region of Upper Scorpius, they are from Garrison (1967). Peculiar stars and extremely rapid rotators have been excluded and the $V$ magnitudes of double stars have been corrected. For the remaining stars in each cluster, a mean absolute magnitude was determined at each spectral type. The internal errors in the resultant composite diagram are quite small, as can be seen from the degree of agreement of the lines; when the main sequences are compared over the entire range where they overlap, it is obvious that a shift of a few tenths of a magnitude would result in a poorer fit.

In the area of overlap, there are no significant differences in rotation or in metal abundance. The suggestion was made yesterday that differences in metal abundance would cause a shift in the HR diagram. This is true for colours, but not for carefully determined spectal types. In the two dimensional MK system, differences in metal abundance would be apparent as peculiarities because the types obtained from the hydrogen lines, from the calcium lines and from the metallic lines would then not be consistent. Thus it is unlikely that a star would be misclassified earlier or later because of an abundance difference.

If it is assumed that the distance modulus of the Hyades is $3 \mathrm{~m} .0$, then the distance modulus of the $\alpha$ Persei cluster is 6.1 and that for the Inner Region of Upper Scorpius is 6.2. In the discussion yesterday (see page 40 ), these values were compared with the values obtained by the convergent point method.

One other way in which this composite main sequence can be tested is by means of the absolute magnitudes for stars with large parallaxes. All main sequence stars with large parallaxes fall near this composite main sequence with no systematic differences, indicating a high degree of consistency over a wide range of spectral types.

Support through the National Research Council of Canada is gratefully acknowledged.

\section{References}

Garrison, R. F.: 1967, Astrophys. J. 147, 1003.

Klare, G. and Szeidl, B.: 1966, Veröffentl. Landessternwarte Heidelberg-Koenigstuhl 18, 9.

Morgan, W. W. and Hiltner, W. A.: 1965 Astrophys. J. 141, 177.

Morgan, W. W., Hiltner, W. A., and Garrison, R. F.: 1971, Astron. J. 76, 242. 